\title{
Seronegative chronic polyarthritis: clinical and serological correlates
}

\author{
J. L. RASKER*, P. DAVIS, AND P. A. BACON \\ From the Royal National Hospital for Rheumatic Diseases, Bath
}

SUMMARY Twenty-seven patients with chronic, active, seronegative polyarthritis were assessed clinically and serologically by accepted criteria for evidence of connective tissue diseases. The patients were divided into 2 groups, 13 positive for fluorescent antinuclear antibody (FANA) and 14 that were negative. All but 1 patient fulfilled the clinical criteria for the diagnosis of definite rheumatoid arthritis. Four patients ( 2 in each group) fulfilled the preliminary criteria for the classification of systemic lupus erythematosus. Antiperinuclear factor was positive in 5 of the FANA-negative patients, consistent with a diagnosis of rheumatoid arthritis. By contrast, this factor was absent in all the FANA-positive patients. Positive DNA binding was found in 7 of the FANA-positive patients, in high titre in 4, consistent with a diagnosis of 'minimal lupus' with predominantly 'unisystem' disease. DNA binding was positive in low titre in 2 of the FANA-negative patients. Antibodies to extractable nuclear antigens (ENA) were found in 4 patients with a positive FANA and in only 1 FANA-negative patient. The current clinical criteria showed poor discriminatory value in classifying cases of seronegative polyarthritis, which may represent mild cases of other connective tissue diseaseo presenting with synovitis in the absence of extra-articular disease manifestations.

Seronegative chronic polyarthritis is a clinically ill-defined diagnosis which is usually made after exclusion of other more clearly defined conditions based on clinical syndrome complexes and often fulfilling accepted clinical criteria. By definition patients with seronegative chronic polyarthritis do not possess IgM rheumatoid factor but may have other clinical features of rheumatoid arthritis (RA) Such patients, whose disease is often called seronegative rheumatoid arthritis, appear to have a more benign prognosis. ${ }^{1}$ Other patients with seronegative chronic polyarthritis may possess fluorescent antinuclear antibodies (FANA) in the absence of other multisystem signs and symptoms to support a diagnosis of systemic lupus erythematosus (SLE). While antinuclear antibodies are found in a variety of connective tissue diseases, they are not a common feature of patients with more clearly defined seronegative arthropathies such as ankylosing spondylitis, psoriatic arthropathy, or colitic arthritis.

Accepted for publication 7 December 1979.

Correspondence to Dr P. Davis, 9-112 Clinical Sciences Building, University of Alberta, Edmonton, Alberta, Canada.

*Current address: Ziekenhuis, Ziekenzorg, Enschede, The Netherlands.
It is therefore unclear whether patients who are negative for IgM rheumatoid factor and positive for FANA have a distinct clinical entity or a more benign variety of such diseases as RA, SLE, mixed connective disease, or progressive systemic sclerosis. The purpose of this study was to assess the presence of antinuclear antibodies in a group of patients with seronegative chronic polyarthritis and to evaluate their significance in relation to other clinical and serological variables.

\section{Patients and methods}

Twenty-seven patients were included in this study. All had polyarthritis of sufficient severity to require admission to the Royal National Hospital for Rheumatic Disease, Bath. All were persistently negative for IgM rheumatoid factor from the outset of their disease. The patients were divided into 2 groups: 13 with a persistently positive FANA and 14 who were negative. All patients were reviewed by one of us (J.J.R.) with special regard to the clinical criteria used for the diagnosis of rheumatoid arthritis, ${ }^{2}$ systemic lupus erythematosus, ${ }^{3}$ and ankylosing spondylitis. ${ }^{4}$ The patients were assessed for functional class by a standard grading. ${ }^{5}$ No 
patients had a history of psoriasis or colitis or clinical features at the time of admission to suggest other connective tissue diseases.

The patients were clinically assessed for evidence of disease activity by the Ritchie score, joint count, and duration of morning stiffness. IgM rheumatoid factor was measured by a commercially available latex fixation test. Sera were tested for antinuclear antibodies by an immunofluorescent technique using mouse liver as substrate at serial dilutions. Antibodies to DNA were measured by a modified Farr technique and a well characterised DNA preparation. ${ }^{6}$ Sera were tested for antiperinuclear factors by a previously described technique ${ }^{7}$ (kindly performed by Dr J. Snijder, Streeklaboratory, Groningen), and antibodies to extractable nuclear antigens (ENA) were detected by a precipitin test (kindly performed by Dr Ian Griffith, Kennedy Institute, London). Radiological assessment of joint disease was obtained from routine $x$-rays of the hands, wrists, and feet. Abnormalities were graded as (0) absent, no abnormalities; (1) mild, periarticular osteoporosis with 1 or 2 cysts; (2) moderate, many cysts with some erosions; or (3) severe, advanced destruction of joints with multiple erosions.

\section{Results}

\section{CLINICAL}

Clinical findings are shown in Table 1. There was no significant difference between the age or sex distribution of those patients who did not have fluorescent antinuclear antibodies and those who did. All patients had evidence of active synovitis, and there was no significant difference in the severity of the disease in the 2 groups as assessed by standard clinical variables. Functional grading was similar in both groups (Table 2). There were no significant differences in the results of haemoglobin, sedimentation rate, routine haematology, and biochemistry in those patients with FANA-positive sera when compared to those without, Extra-articular manifestations were not a prominent feature of either

Table 1 Clinical findings

\begin{tabular}{llll}
\hline & \multicolumn{2}{l}{ Seronegative polyarthritis-IgM RhF negative } \\
\cline { 2 - 4 } & $\begin{array}{l}\text { FANA negative } \\
n=14\end{array}$ & $\begin{array}{l}\text { FANA positive } \\
n=13\end{array}$ & $\begin{array}{l}P \\
\text { (Student's } \\
\text { test) }\end{array}$ \\
\hline & & & \\
\hline Disease duration & & 3.7 & $<0.025$ \\
$\quad$ (years) & 10.1 & 42.1 & $\mathrm{NS}$ \\
ESR (mm/h) & 45.8 & 11.9 & $\mathrm{NS}$ \\
Hb (G\%) & 11.4 & 1.08 & $<0.02$ \\
$X$-ray grading & 1.96 & 2 & $\mathrm{NS}$ \\
Nodules & 3 & & \\
\hline
\end{tabular}

NS $=$ not significant.
Table 2 Functional grading of patients with chronic seronegative polyarthritis

\begin{tabular}{lcl}
\hline Functional grade & FANA-positive & FANA-negative \\
\hline I & 1 & 0 \\
II & 11 & 8 \\
III & 1 & 5 \\
IV & 0 & 1 \\
\hline
\end{tabular}

group, and rheumatoid nodules were found equally in the 2. There was a significant difference between the duration of the disease in the 2 groups. Patients positive for FANA had a mean disease duration of 3.7 years, those negative for FANA $10 \cdot 1$ years. In addition, there was a statistically significant difference in the $x$-ray grading. Those patients positive for FANA having less severe evidence of joint destruction than those without.

\section{CLASSIFICATION}

All but 1 patient fulfilled the ARA criteria for the classification of definite or classical rheumatoid arthritis (Table 3). Two patients in each group fulfilled the American Rheumatism Association's (ARA) preliminary criteria for the classification of SLE. All these 4 patients also fulfilled the criteria for the diagnosis of rheumatoid arthritis. None of these patients had clinical or biochemical evidence of skin, renal, or nervous system disease. No patients fulfilled the New York criteria for the classification of ankylosing spondylitis.

\section{SEROLOGICAL FINDINGS}

There was a marked difference in the prevalence of positive serological tests in the 2 groups (Table 4). Patients with negative antinuclear antibodies had antibodies to native DNA in 2 instances at low

\section{Table 3 Clinical criteria}

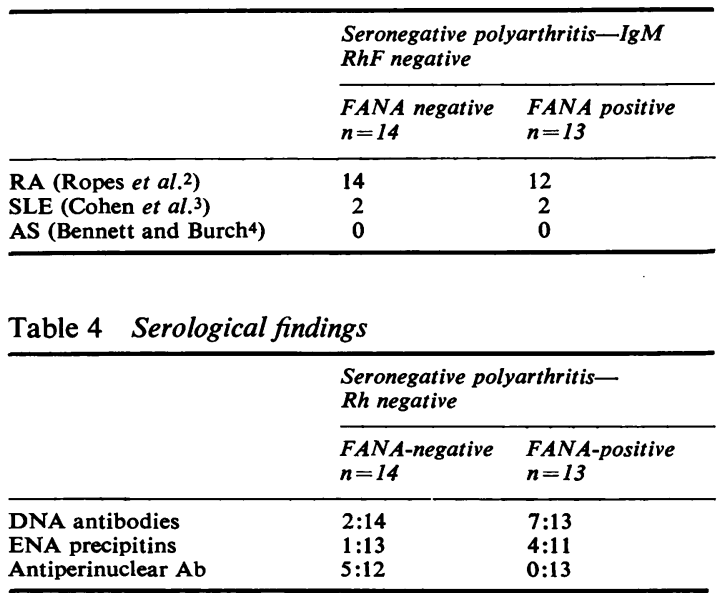


titre, and 1 patient had a positive precipitin to ENA. These patients were not the same as those who fulfilled the ARA preliminary criteria ror the classification of SLE. Antiperinuclear antibody was a feature of patients with a negative FANA, being present in 5 out of 12 patients in contrast to none of the patients with a positive FANA. Seven patients with a positive fluorescent antinuclear antibody test had antibodies to double stranded DNA, and in 4 of these cases this was present in high titre. The highest DNA binding was found in the patients with the highest titre of fluorescent antinuclear antibody, and both patients who fulfilled the ARA preliminary criteria of the classification of SLE had DNA binding. Four patients with a positive FANA had precipitin lines to ENA.

\section{Discussion}

In this study an attempt has been made to delineate subsets of patients with seronegative chronic polyarthritis on the basis of clinical and serologic variables. All the patients had active synovitis, and there were no clinical features to suggest that the patients with positive antinuclear factors were different from those in whom the antinuclear factor was negative. The major clinical difference in the 2 groups was a milder radiographic picture of the patients with a positive antinuclear antibody, but this may reflect the difference in duration of diseases, which was significantly shorter in the patients with a positive FANA than those in whom it was negative. Long-term studies will be necessary to determine whether patients positive for FANA have a milder course than those in whom it is negative.

The use of accepted criteria for the diagnosis of specific disease entities proved not to be useful in defining different subsets in our patients. All but 1 patient fulfilled the ARA criteria for the diagnosis of definite or classical rheumatoid arthritis, but 4 of these patients, 2 in each group, also fulfilled the criteria for the diagnosis of SLE. One patient fulfilled neither. No patient fulfilled the criteria for the diagnosis of ankylosing spondylitis. Our study illustrates the limitations of such criteria when applied to patients with seronegative chronic polyarthritis, in whom other specific diseases have been excluded. It would seem reasonable to regard those patients negative for rheumatoid factor and for FANA as being patients with mild rheumatoid arthritis. Although these patients in our study were not compared to patients with rheumatoid-factorpositive rheumatoid disease, the duration of disease in our patients associated with their relatively good functional grade and mild radiological changes would certainly suggest that they have a more benign prognosis. Two patients in this group had slightly raised DNA binding, but were not the same 2 patients who fulfilled the ARA preliminary criteria for the diagnosis of SLE. This finding in patients negative for FANA and lacking clinical features of the disease is difficult to explain, but may reflect binding to single-stranded determinants in the antigen used, which is known to contain $15 \%$ single-stranded DNA, rather than antibody binding to double-stranded determinants, which would have diagnostic significance for SLE. ${ }^{8}$ This may also account for previous reports of antibodies to n.DNA in other connective tissue diseases. The presence of antiperinuclear factor in some of our patients negative for antinuclear antibody (ANA) would also suggest that these patients have RA, as this factor is found almost exclusively in this condition. ${ }^{910}$

Classification of patients with ANA-positive, seronegative chronic polyarthritis is less clear. Certainly at least 2 patients in this study fulfilled the American Rheumatism Association's criteria for the classification of SLE in the presence of a positive FANA and raised DNA binding, and would therefore appear to have previously unsuspected SLE. One patient, a man of 58, had a 5-year history of progressively active synovitis with minimal radiographic changes and no extra-articular manifestations of his disease. The other, a woman of 48 , had a nonerosive arthritis with nodules. In this case there was a previous history of convulsions and pleuritis but no evidence of renal disease. One further patient had a strongly positive FANA, high DNA binding in the presence of arthritis alone, and may therefore also have SLE with clinical manifestations affecting only 1 system at present.

Moderately elevated levels of DNA binding were seen in other patients in the presence of a positive FANA and the absence of clinical features to suggest the diagnosis of SLE. These cases may represent mild or benign SLE with features similar to those described by Go and Lockshin ${ }^{11}$ and Linn et al. ${ }^{12}$ Despite minor differences in patient selection and grouping, many of the features described in these studies are similar to those in ours. However, Lin and colleagues found fewer nodules and less destructive disease in their FANA-negative group in contrast to our findings. This difference may be due to the shorter disease duration in our FANApositive patients. In addition the patients in our study had features clinically more similar to rheumatoid arthritis than SLE, with a low incidence of extra-articular lesions. These findings are in contrast particularly with those in the patients reported by Go and Lockshin, in whom 10 of 17 had multisystem disease and features both clinically and 
serologically of SLE. Our patients would therefore represent mild cases of unisystem disease with serological evidence of SLE. Certainly cases of serologically active, clinically quiescent disease have been reported and may resolve spontaneously. ${ }^{13}$ In patients such as these the use of a battery of physiological and pathological tests may reveal more extensive disease than is clinically apparent. ${ }^{14}$

The presence of precipitins to ENA in 4 of the patients with a positive FANA raises the possibility that these patients may have mixed connective tissue disease. A nondeforming arthropathy has been well defined in this condition and may not necessarily be seen in association with other systemic features of the disease. ${ }^{15}$ This would be consistent with the relatively benign nature of the patients' disease in the absence of severe radiographic change. Some patients with seronegative chronic polyarthritis and a positive FANA had no other serological abnormality, resulting in a group of patients in whom clinical criteria and serological tests failed to help determine a specific diagnosis.

We conclude from study of 27 patients with seronegative chronic polyarthritis that currently acceptable clinical criteria are of limited value in attempting to classify these patients. A serological battery of tests may be of more value, with the presence or absence of a positive test for FANA being a useful screening test. In some cases these tests may be useful for serological classification, but in our cases they did not necessarily reflect different patterns of disease as assessed clinically.

\section{References}

1 Masi A T, Maldonado-Cocco J A, Kaplan S B, Feigenbaum $S$ L, Chandler $R$ W. Prospective study of the early course of rheumatoid arthritis in young adults: comparison of patients with and without rheumatoid factor positivity at entry and identification of variables correlating with outcome. Semin Arthritis Rheum 1976; 5: 299-326.

2 Ropes M W, Bennett G A, Cobb S, Jacox R, Jessar R A. 1958 revision of diagnostic criteria for rheumatoid arthritis. Ann Rheum Dis 1959; 18: 49-53.

3 Cohen A S, Reynolds W E, Franklin E C, et al. Preliminary criteria for the classification of SLE. Bull Rheum Dis $1971 ; 21$ : 643-8.

4 Bennett R H J, Burch T A. New York symposium of population studies in the rheumatic disease-new diagnostic criteria. Bull Rheum Dis 1976; 17: 453-8.

5 Steinbrocker O, Traeget C H, Batterman R C. Therapeutic criteria in rheumatoid arthritis. JAMA 1949; 140: 659-62.

6 Davis P, Burrington M, Morgan A R, Saetre A. Evaluation of a standardised assay for the measurement of antibodies to double stranded (native) DNA. J Clin Pathol 1977; 30: 827-30.

7 Nienhuis R L F, Mandema E, Smids C. A new serum factor in patients with RA: the antiperinuclear factor. Ann Rheum Dis 1964; 23: 302-5.

8 Davis P, Burrington M, Russell A S, Morgan A R. Analysis of DNA structure by hydroxyapatite columns and ethidium bromide fluorescence techniques. Arthritis Rheum 1978; 21 : 407-13.

9 Nienhuis R L F. Evaluatie von de antiperinucleaire factor (APF). Ned Tijdschr Geneeskd 1973; 117: 1561-5.

10 Smit J W, Aay C, Lakroots W R, Feldkamp-Vroom T, Feldkamp T E W. Antiperinucleaire factor. Med Tijdschr Geneeskd 1976; 120: 1147-8.

11 Go T, Lockshin M. Latex negative ANA positive erosive arthritis. Prognosis more like SLE than RA. Arthritis Rheum 1975; 18: 401-2.

12 Linn J E, Hardin J G, Halla J T. A controlled study of ANA + and RF-arthritis. Arthritis Rheum 1978; 21: 645-51.

13 Lightfoot R W, Hughes G R V. Significance of persisting serologic abnormalities in SLE. Arthritis Rheum 1976; 19: $837-43$

14 Edmonds J P, Hughes G R V. Subclinical involvement and serological abnormalities in "minimal lupus". Scand J Rheumatol 1975; Suppl. 8: 42/04.

15 Halla J T, Hardin J G. Clinical features of the arthritis of mixed connective tissue disease. Arthritis Rheum $1978 ; 21$ : 497-503. 